\title{
TEAD4 Induced Positive Feedback Activation of E2R/YAP1 Axis and Promoted Epithelial- mesenchymal Transition through Up-regulating TWIST1 Directly in HCC
}

Lin Liu the first Affiliated hospital of Xi'an Jiaotong University

Mi Ke

The First Affiliated Hospital of Xi'an Jiaotong University

Chuzhi Shang

the first Affiliated Hospital of Xi'an Jiaotong University

Yufang Liu

the First Affiliated Hospital of Xi'an Jiaotong University

\section{Cong Wang}

the First Affiliated Hospital of Xi'an Jiaotong University

Xin Zheng ( $\square$ zhengxin@xjtufh.edu.cn )

the First Affiliated Hospital of Xi'an Jiaotong University https://orcid.org/0000-0002-7306-9688

\section{Research Article}

Keywords: TEAD4, E2R, TWIST1, EMT, HCC

Posted Date: July 1st, 2021

DOl: https://doi.org/10.21203/rs.3.rs-602611/v1

License: (c) (i) This work is licensed under a Creative Commons Attribution 4.0 International License.

Read Full License 


\section{Abstract}

Background: Great advance has been achieved in the investigation of Hippo signaling on mediating carcinogenesis. However, the underlying mechanism that YAP1/TEAD4 signaling regulated hepatocellular carcinoma (HCC) progression have not been completely determined. This investigation aimed to determine the mechanism of activation of YAP1/TEAD4 pathway and its regulatory effect on hepatocarcinogenesis.

Methods: The TCGA database and immunohistochemical (IHC) analyses about HCC samples were carried out to analyze the expression of TEAD4 and its correlation with HCC prognosis. CCK-8, wound healing assay and transwell chamber with Matrigel were used to examine the role of TEAD4 on cell proliferation, migration and invasion capacities. In vivo imaging system was used to observe the tumor formation in SCID mouse model. The mechanistic investigation was conducted with functional studies, Western immunoblotting, Luciferase reporter assay, chromatin immunoprecipitation.

Results: Analysis of HCC samples revealed that TEAD4 was up-regulated in HCC tissues compared to adjacent liver tissues, and its overexpression in HCC was significantly associated with worse prognosis, high serum AFP level, larger tumor size, PVTT, multiple tumor lesions, and microvascular invasion. Enforced expression of TEAD4 in HCC cell lines promoted the proliferation and growth of HCC cells in vitro and in vivo. The further functional studies showed that TEAD4 increased TWIST1 expression directly in the binding-promoter manner and then enhanced the migration and invasion of HCC cells via inducing epithelial-to-mesenchymal transition (EMT).TWIST1 was found to enhance E2R expression and consequently formed a positive feedback loop to activate the YAP1/TEAD4/TWIST1 axis.

Conclusion: This investigation provided the functional and mechanistic basis for identifying the E2R/YAP1/TEAD4/TWIST axis as the oncogenic factor which inducing EMT and then accelerated HCC growth and invasion.

\section{Introduction}

Hepatocellular carcinoma (HCC) is the most common kind of primary liver cancer (more than $80 \%$ ), which has been the fourth leading cause of cancer-related deaths all through the world[1]. Although there have been advancements in diagnostic and treatment approaches about HCC, the outcome of HCC remains unsatisfied due to the highly metastatic and aggressive biological features of $\mathrm{HCC}[2]$. In recent years, the results of randomized clinical trials about Tyrosine kinase inhibitors (TKIs), antiangiogenetic agents and immunotherapy with checkpoint inhibitors have led to a paradigm change in the scenario for the therapy of HCC. These systemic treatments synergizing with locoregional therapies including radiofrequency ablation, transarterial chemoembolisation, external beam radiotherapy did not only improved the prognosis of advanced HCC, but also worked as the effective tumor down-staging treatment, which made more HCC patients at the advanced stage suitable to receive curative liver resection[3, 4]. However, the respond to these systemic therapies was still unpredictable, and HCC patients suffered from favorable 
outcome constitute only a subset of the overall population. Hence, it remains essential to discover the molecular mechanism about carcinogenesis and metastasis for developing the novel efficient therapeutic strategy for HCC.

YAP1/TEAD4 pathway (Hippo pathway) was first found as a key mediator of organ size in Drosophila melanogaster. Briefly, when Hippo pathway was activated, MSTs/SAV1 complex phosphorylated LATSs/MOB1 complex, and consequently repressed phosphorylation of YAP1, which inhibited ubiquitination and proteasomal degradation of YAP1 $[5,6]$. Upon repressed phosphorylation, more YAP protein translocated to cell nucleus, and then interacted with TEAD4 to control the transcription of various its downstream genes regulating tumor progression[7, 8]. Currently, TEAD4 was demonstrated to be aberrantly over-expressed in a variety of cancers including head-neck squamous cell carcinoma[9], bladder cancer[10], gastric cancer[11], colorectal cancer[12], ovarian cancer[13], and pancreatic cancer[14]. As to HCC, previous studies showed that increased YAP/TAZ activity was found about $60 \%$ of HCC samples[15-17]. The expression of YAP has been found to be control via various molecular mechanisms, including both transcriptional and epigenetic regulation[18, 19]. It has been unclear about the mechanism of activation of YAP1/TEAD4 signaling in HCC and association between TEAD4 and HCC prognosis.

Here, we showed that TEAD4 expression was increased significantly in HCC tissues compared with adjacent liver tissues, and higher TEAD4 expression in tumor tissues was associated positively with the unfavorable prognosis. TEAD4 was found to induce EMT phenotype of HCC cells via promoting TWIST1 expression directly by bound with promoter. Consequently, TWIST1 activated YAP1/TEAD4 pathway through up-regulating E2R, a member of GPCRs. And over-expression of TEAD4 accelerated the growth and metastasis of HCC in vitro and in vivo via inducing TWIST1-dependent EMT phenotype. The above findings about positive feedback control of E2R/YAP1/TEAD4 signaling by TWIST1 provided the novel insights into potential approaches in HCC therapy.

\section{Methods}

\section{HCC specimens and reagents}

This investigation has been approved by the Ethical Committee of the First Affiliated Hospital of Xian Jiaotong University. One hundred and thirty HCC samples were obtained from the Department of Hepatobiliary Surgery, the First Affiliated Hospital of Xian Jiaotong University with written informed consent from the patients. Rabbit anti-TEAD4 (Catalog. No.: ab197589), rabbit anti-YAP1 (Catalog. No.: ab205270), p-YAP1 (phospho S127, Catalog. No.: ab76252), Rabbit anti-E-cadherin (Catalog. No.: ab40772), Rabbit anti-N-cadherin (Catalog. No.: ab76011), mouse anti-Vimentin (Catalog. No.: ab20346), mouse anti-TWIST1 (Catalog. No.: ab175430), and mouse anti-E2R (Catalog. No.: ab233741) were acquired from Abcam (Cambridge, UK). Rabbit anti- $\beta$-actin (Catalog. No.: 8457) was purchased from Cell Signaling Technology (Danvers, USA). Both HRP-linked goat anti-rabbit antibody (Catalog. No.: ab7090) and HRP-linked goat anti-mouse antibody (Catalog. No.: ab97040) were procured from Abcam 
(Cambridge, UK). TEAD4-targeted siRNAs (TEAD4 siRNA, Catalog. No.: sc-96187) and scramble siRNAs (Scr siRNA, Catalog. No.: sc-37007) were obtained from Santa Cruz Biotechnology (Dallas, USA). The sequences of siRNA against E2R (Thrombin R siRNA, Catalog. No.: sc-36663) and scramble siRNAs (Catalog. No.: sc-37007) were from Santa Cruz Biotechnology (Dallas, USA). Human TEAD4-expressing plasmid (Catalog. No.: RC219686) and TWIST1-expressing plasmid (Catalog. No.: RC202920) were purchased from OriGene Technologies, Inc (Rockville, USA). Luciferase reporter plasmids containing the wild type (WT) or mutant (mut) TEAD4-binding sites were synthesized by GENECHEM CO. (Shanghai, China).

\section{HCC cell lines and stable transfection}

HCC cell lines including Hep3B, PLC/PRF/5, BEL7402, HCCLM3 and HepG2 cells were directly obtained from American Type Culture Collection (Manassas, VA, USA) which were passaged less than 6 months in our laboratory. Huh7 cell line was a gift from Prof. Kefeng Dou (Department of Hepatobiliary, Xijing Hospital of AIR FORCE MEDICAL UNIVERSITY). Both MHCC97h and SMMC7721 cells were purchased from Cell Bank of Chinese Academy of Sciences (Shanghai, China). All cells were cultured in DMEM medium with $10 \% \mathrm{FBS}$ in a humidified cell incubator at $37^{\circ} \mathrm{C}$ with an atmosphere of $5 \% \mathrm{CO} 2$.

The transfection of siRNAs against TEAD4, E2R and scramble siRNA were performed using siRNA Transfection Reagent recommended by Santa Cruz Biotechnology (Catalog. No.: sc-29528, Dallas, USA) according to the manufacturer's protocol. For the generation of stable transfection clones, Huh7 or Hep3B cells were cultured respectively onto six-well plates to reaching $80 \%$ confluence. HCC cells were transfected with $4.0 \mu$ g plasmids using FuGENE@6 transfection reagent (Catalog. No.: E2691, Promega, Madison, USA). After 48h, HCC cells were harvested, selected in DMEM with containing $600 \mu \mathrm{g} / \mathrm{mL}$ Neomycin for 2 weeks and then subjected to limited dilution to isolate and expand the stable target gene transfected HCC cells. The selected transfection clones were subsequently cultured under selective conditions.

\section{Quantitative real-time polymerase chain reaction (qRT-PCR)}

The qRT-PCR assays were performed as described briefly as following: total RNA was extracted from HCC cells using TRIzol ${ }^{\circledR}$ reagent (Invitrogen, CA, USA) and CDNA was reverse-transcribed using the PrimeScript ${ }^{\circledR}$ RT Reagent Kit (TaKaRa, China) following the instructions. The qRT-PCR assay was run with the SYBR® Premix Ex Taq ${ }^{\text {TM }}$ Kit. GAPDH was used as the internal control. The primers involved in this study was listed at Table 1.

\section{Cell viability, migration and invasion}

Cell Counting kit-8 (CCK-8) was conducted to measure cell viability. Briefly, HCC cells were seeded into 96well plates at a density of $3 \times 10^{3}$ cells per well in $10 \mu \mathrm{L}$ of 
DMEM medium with fetal bovine serum (FBS) and grown for $24 \mathrm{~h} .10 \mu \mathrm{L}$ of CCK-8 reagent was added per well, and $\mathrm{HCC}$ cells were incubated for $2 \mathrm{~h}$. The results were obtained with the Termo Fisher Scientifc microplate reader at $450 \mathrm{~nm}$ (A450).

Cell migration was measured by wound healing assays. Briefly, HCC cells $\left(1 \times 10^{6}\right.$ cells $)$ were plated in 6well plates and cultured for $24 \mathrm{~h}$. The wound was incised in the central area of the confluent culture. The detached HCC cells were washed and DMEM medium with $10 \%$ FBS was used to culture HCC cells. Pictures were taken of the wounded area 0 and $48 \mathrm{~h}$ later using the digital camera.

Transwell chamber with Matrigel assays were performed to assess invasion abilities. HCC (cells $1 \times 10^{5}$ cells) were seeded into 24-well transwell filters (Corning, NY, USA) with Matrigel (BD Biosciences, USA) covered. DMEM medium with $10 \%$ FBS was added into the lower chamber. HCC cells were grown for $24 \mathrm{~h}$, fixed and stained with $0.1 \%$ crystal violet and counted under microscopy.

\section{Western immunoblotting}

HCC cells were lysed in RIPA buffer with PMSF. Protein samples were electrophoresed on polyacrylamide gel electrophoresis (PAGE) and transferred onto Nitrocellulose membrane (NC). Protein on the membrane was block overnight at $4^{\circ} \mathrm{C}$ using blocking buffer (nonfat dried milk diluted in Tris-buffered saline containing $0.1 \%$ Tween-20 buffer (TBST)), and incubated with the primary antibodies, respectively. Then, protein samples on the NC membranes were washed twice with TBST buffer and incubated with the relative secondary antibodies. The immunoreactive protein bands were detected using the HyGLO HRP detection kit from Denville (NJ, USA). $\beta$-actin was used as the internal control.

\section{Immunohistochemistry}

Clinical samples harvested from HCC patients or HCC xenografts from mice were obtained and fixed in formalin for paraffin sectioning. The immunohistochemistry staining was carried out as described previously[20]. Briefly, tissue slides (4-mm thick) were de-paraffinized with xylene and rehydrated with graded alcohols. Endogenous peroxidase activity was blocked for $2 \mathrm{~h}$ with methanol solution containing $0.3 \%$ hydrogen peroxide. Then, antigens were retrieved in citrate buffer and blocked overnight at $4^{\circ} \mathrm{C}$. After washing with PBS buffer, tissue samples were incubated with the respective primary antibodies directed against TEAD4 (Catalog. No.: ab97460, dilution: 1:100) and PCNA (Catalog. No.: ab29, dilution: 1:10000) at $4^{\circ} \mathrm{C}$ overnight. Tissue sections were rinsed with PBS buffer and then incubated with the relevant secondary antibodies, detected with diaminobenzidine and counterstained with hematoxylin.

To examine the $\mathrm{IHC}$ staining immunoreactivity, we tested the staining intensity and the ratio of specifically positive staining cells. The staining of yellowish or brownish in cytoplasm or nucleus was divided into the following scale: 0 , none; 1 , weak; 2 , moderate; 3 , strong. The ratios of specifically positive staining cell number/total cell number was classified using the following grades: $0(<5 \%), 1(6 \%-25 \%), 2$ $(26 \%-50 \%), 3(51 \%-75 \%)$, and 4 (N75\%). These two parameters were multiplied to get the score of IHC staining: 0 - 1 were negative results and $\geq 2$ was considered as positive staining. 


\section{Chromatin immunoprecipitation (ChIP)}

ChIP was conducted with EZ-Magna ChIP kit (Millipore, Billerica, MA, USA) according to the recommended protocol to detect the binding of TEAD4 to the TWIST1 promoter and TWIST1 to the E2R promoter. Briefly, crosslinking was carried out with $1 \%$ formaldehyde, and the HCC cells were washed, lysed in SDS buffer and sonicated to prepare the DNA fragment sample using a sonication apparatus. The lysates were immunoprecipitated overnight with gentle rotation at $4^{\circ} \mathrm{C}$ using the antibodies against TEAD4 or TWIST1. DNA extraction was conducted with a Qiagen Purification kit and PCR assessment was carried out using Ultra HiFidelity PCR Kit. The primers were used to amplify the TWIST1 promoter, Forward primer 5- GC T $C \top G A \mathbb{C} C \top C G G T C \mathrm{~T}$ - 3, Reverse primer 5

- $A \mathbb{C} A T G G \forall T G T G C \forall \forall T G C T$ - 3; the primers amplifying E2R promoter were listed in Fig.7D.

\section{Luciferase Reporter Gene Assay}

The determined protein-binding sites were inserted into the Renilla luciferase plasmid, respectively. The recombinant luciferase plasmid was transfected into HCC cells, and then luciferase activity was assessed by the Dual-Luciferase Reporter Assay System (Promega, USA).

\section{Growth and metastasis assays in vivo}

All animal experiments were conducted according to the "Guide for the Care and Use of Laboratory Animals" from the National Academy of Sciences and published by the National Institutes of Health (NIH publication 86-23 revised 1985) and approved by Experimental Animal Care and Use Committee of Xian Jiaotong University (XJTU1AF2015LSL-024). The BALB/c nude mice (four weeks old, male) were purchased from the animal center of Xian Jiaotong University. For HCC xenograft growth assay, 10 nude mice were randomly divided into two groups, and Huh7 TEAD 4 cells $\left(1 \times 10^{7}\right)$ were subcutaneously injected into the nude mice to be Huh7 TEAD4 group, while Huh7 Vector cells $\left(1 \times 10^{7}\right)$ were implanted into each nude mouse subcutaneously to be Huh7 Vector group. The length and width of HCC xenografts were measured with a digital Vernier calliper every week and the volume of HCC xenografts was observed using the following formula: volume $=A \times B^{2} \times 0.52$ ( $A$, length; $B$, width). For HCC xenograft metastasis analysis, Huh7 TEAD4 cells $\left(1 \times 10^{7}\right)$ (transfection with TEAD4 expressing GV492 gcGFP Lentivirus) or Huh7 Vector cells $\left(1 \times 10^{7}\right)$ were injected into the nude mice via the tail vein. Bioluminescence was observed by IVIS@ Lumina II system 4 weeks after injection of HCC cells.

\section{Statistical analysis}

All data were analyzed with GraphPad Prism 8.0 Software (GraphPad Inc.). The parameterized variables were examined by Student's t-test or Mann-Whitney $U$ test and the results are presented as the mean $\pm S D$. Comparison of Kaplan-Meier survival curves was tested by log-rank test. $P$ value $<0.05$ was considered statistically significant.

\section{Results}




\section{Up-regulation of TEAD4 was correlated with poor prognosis of HCC}

To determine the expression profile of TEAD4 in HCC, we analyzed TEAD4 expression in HCC and matched adjacent liver tissues at the mRNA level, and found that TEAD4 mRNA was dramatically downregulated in HCC tissues (Fig.1A). Subsequently, immunohistochemistry (IHC) staining showed that there was more TEAD4 protein expression found in tumor tissues than adjacent liver tissues, which was confirmed by compared IHC scores by Mann-Whitney U test (Fig.1B). And TEAD4 protein located in cell nucleus mainly and detected 89 (68.5\%) out of the 130 HCC tissues compared with $36(27.7 \%)$ out of the 130 adjacent liver tissues. This result also verified that TEAD4 expression was increased in HCC tissues. Next, the association between TEAD4 expression and clinical characteristics of HCC cases. As presented in Table 2, high TEAD4 expression in HCC tissues was positively related with high serum AFP level $(\mathrm{P}=$ 0.002), larger tumor size $(P=0.001)$, PVTT $(P=0.021)$, multiple tumor lesions $(P=0.002)$, and microvascular invasion (MVI, $\mathrm{P}<0.001) .98 \mathrm{HCC}$ patients with the follow-up information were divided into High TEAD4 and Low TEAD4 group using the ratio of TEAD4 expression in HCC/adjacent liver tissues as the cut-off value, and it was found that patients from High TEAD4 group obtained shorter post-surgical overall survival by comparison with Kaplan-Meier curve (HR $=3.282,95 \% \mathrm{Cl}(1.925$ to 5.594$), \mathrm{P}<0.001$, Fig.1C). The median overall survival time of High TEAD4 group was 19.75 months, while one of Low TEAD4 group was 89.22 months. Univariate analysis showed that liver cirrhosis, advanced TMN staging, portal vein invasion (PVTT), multiple tumor lesions and higher TEAD4 expression in tumor tissues were the poor prognostic factors, while multivariate Cox regression analysis confirmed liver cirrhosis, PVTT, multiple tumor lesions, MVI and higher TEAD4 expression in tumor tissues as the independent predictive factors after surgery (Table 3). These results were further confirmed by data from the Cancer Genome Atlas (TCGA) database. As shown in Fig.1D, TEAD4 mRNA was found increased significantly $(P<0.001)$ in $371 \mathrm{HCC}$ tissues compared with 50 normal liver tissues. And data from TCGA database verified that HCC patients with high TEAD4 expression (High expression group) suffered from worse prognosis than those with low/medium TEAD4 expression (Low/Medium-expression group, Fig.1E, $P=0.049$ ).

\section{TEAD4 accelerated HCC growth in vitro and in vivo}

To determine the functional roles of TEAD4 in HCC progression, the expression of TEAD4 was examined in HCC cell lines (Fig.2A). Huh7 and Hep3B cells were chosen to establish TEAD4-overexpressing HCC models, whereas MHCC97h cells was selected as TEAD4-silencing HCC cell model. Both qRT-PCR and Western immunoblotting assays were carried out to verify transfection efficiency (Fig.2B). As shown in Fig.2C, CCK-8 assay indicated that overexpression of TEAD4 enhanced the proliferation ability of HCC cells, while silencing TEAD4 obtained the opposite results in MHCC97h cells. BrdU assay also was conducted to examine cell proliferation capacity of HCC cells and found that enhanced expression of TEAD4 increased proliferation of both Huh7 (Suppl.Fig.1 A) and Hep3B cells (Suppl.Fig.1 B) dramatically, while inhibiting proliferation ability of MHCC97h cells after knockdown of TEAD4 (Suppl.Fig.1 C).

To further figure out the oncogenic function of TEAD4 in HCC, we carried out in vivo experiments with nude mouse subcutaneous tumor models. Enforced expression of TEAD4 in Huh7 cells was found to 
increase the growth of HCC xenografts (Fig.2D). And IHC staining assay confirmed that PCNA, a marker of proliferation, was detected along with the elevated expression of TEAD4 (Fig.2E), as well. These revealed that TEAD4 accelerated HCC growth in vitro and in vivo.

\section{TEAD4 enhanced migration capacities of HCC cells and accelerated metastasis in vivo}

Next, we attempted to determine the effect of TEAD4 on HCC metastasis. And we carried out wound healing assay and noticed that enforced expression of TEAD4 increased the speed of wound closure of both Huh7 and Hep3B cells (Fig.3A). As assessed by Transwell invasion assay with Matrigel matrix, it was found that TEAD4 enhanced invasion capacity of Huh7 and Hep3B cells (Fig.3B). Knockdown of TEAD4 in MHCC97h cells showed the opposite effect (Fig.3C). Besides, Western immunoblotting assay revealed that ectopic expression of TEAD4 in Huh7 cells remarkably increased the expression of mesenchymal makers $\mathrm{N}$-cadherin and Vimentin and suppressed epithelial marker E-cadherin, while knockdown of TEAD4 in MHCC97h cells resulted in the opposite changes in epithelial and mesenchymal markers (Fig.4A). Additionally, EMT transcriptional factor TWIST1 expression was also up-regulated by overexpression of TEAD4 in Huh7 cells and inhibited by silencing TEAD4 in MHCC97h cells (Fig.4A). And analysis of TCGA database confirmed that there was significantly positive correlation between TEAD4 and TWIST1 at the level of mRNA in HCC tissues (Fig.4B). With the help of TEAD4 expressing GV492 gcGFP Lentivirus, we perform the in vivo bioluminescence imaging assay and found that overexpression of TEAD4 in Huh7 cells promoted metastasis of HCC cells in vivo significantly (Fig.4C). These suggested that TEAD4 promoted metastatic ability of HCC cells via inducing EMT.

\section{TEAD4 up-regulated TWIST1 expression in HCC cells via binding with its promoter directly}

Typically, transcription factors (TFs) have been found involved closely in the activation of the transcription of its down-stream genes. Due to the positive correlation between TEAD4 and TWIST1 found in HCC mentioned above in this study, we hypothesized that

TEAD4 was involved in the transcription that leads to TWIST1 overexpression in HCC. To verify this hypothesis, we made analysis of TWIST1 promoter using the JASPAR algorithm and observed 1 potential TEAD4-binding site region (Fig.5A). The primes against the -550 -351 bp of TWIST1 promoter were designed for chromatin immunoprecipitation (ChIP) assessment as following: Forward primer 5 - GC T $C$ T GAC C T CGGTC T - 3, Reverse primer 5- $A \mathbb{C} A T G G \forall T G T G C \forall \forall T G C T$ - 3. Furthermore, ChIP assay results revealed that TWIST1 gene promoter was directly immunoprecipitated with anti-TEAD4 antibodies and there were significantly more fractions of TWIST1 promoter bound with TEAD4 in Huh7 TEAD4 cells than Huh7 Vector cells (Fig.5B). Luciferase reporter plasmids containing the wild type (WT) or mutant (mut) TEAD4-binding sites were used for TEAD4 promoter activity, respectively. And as assessed by luciferase reporter assays, it was found that TEAD4 protein was no longer able to induce the activity of the TWIST1 promoter in a reporter construct lacking the TEAD4 bindings sites (Fig.5C). 
Several evidences showed that G protein-coupled receptors (GPCRs) modulated the phosphorylation states of YAP protein and activated Hippo signaling[21, 22]. We attempt to test the hypothesis that TWIST1 increased one member of GPCRs family and feedback activated Hippo/YAP/TEAD4 signaling. As shown in Fig.6A, The analysis of HCC cohort from the TCGA database revealed that TWIST1 mRNA expression was positively correlated with GPCR E2R (protease-activated receptor 1, also called as PAR1, $r$ $=0.561, P<0.001)$ in HCC tissues $(n=374)$. And E2R mRNA expression was also positively associated with YAP1 ( $r=0.434, P<0.001$, Fig.6B) and TEAD4 ( $r=0.357, P<0.001$, Fig.6C). To investigate the causal regulatory effect of TWIST1 in E2R expression, we built stable TWIST1 expressing clones and control clones in Huh7 cells (Fig.6D). As shown in Fig.6D, TWIST1 overexpression increased expression of E2R, YAP1 and TEAD4, while decreasing YAP1 phosphorylation in Huh7 cells. And loss of E2R expression up-regulated phosphorylation of YAP1, and repressed the expression of YAP1 and TEAD4 in both Huh7 TWIST1 cells and MHCC97h cells (Fig.6E), which indicated that E2R was critical to activation of YAP1/TEAD4 signaling in HCC cells.

Given the positive induction of E2R expression of TWIST1, we attempted to figure out whether TWIST1 mediated E2R expression directly. We noticed that E2R promoter contained 10 potential TWIST1-binding E-boxes (CANNTG) from -1346 bp to transcription start site (TSS, Fig.7A). According to the location of potential TWIST1-binding E-boxes, we divided the E2R promoter into 3 potential TWIST1 protein-binding fractions: Fraction 1, -1999 bp -1756 bp; Fraction 2, -1638 bp -1456 bp; Fraction 3, -668 bp -425 bp. Furthermore, we cloned the E2R promoter (Luc 1) and made deletion mutants of promoter luciferase constructs according to the location of the 3 potential TWIST1 protein-binding fractions (Fig.7B). The mutant E2R promoter without Fraction 1 was rebuilt into the Renilla luciferase vector as Luc 2. The mutant E2R promoter without Fraction 2 was reconstructed into the Renilla luciferase vector as Luc 3. The mutant E2R promoter without Fraction 3 was reconstructed into the Renilla luciferase vector as Luc 4. As shown in Fig.7C, when transfecting the full-length E2R promoter (Luc 1) in Huh7 cells, overexpression of TWIST1 activated the E2R promoter activity dramatically. And Luc 2 transfection leaded to significant loss of E2R promoter activity. Interestingly, Luc 3 transfection also decreased the activity of E2R promoter clearly, while Luc 4 still remained the relative higher E2R reporter activity to respond to TWIST1 overexpression. These results indicated strongly that both Fraction 1 and Fraction 2 were critical to the activation of E2R transcription driven by TWIST1. To verify the binding between TWIST1 protein and E2R promoter, ChIP assay was carried out by different primers against Fraction 1 , Fraction 2 and Fraction 3, respectively (Fig.7D). It was found that more both Fraction 1 and Fraction 2 of E2R promoter was bound with TWIST1 protein in Huh7 TWIST1 cells compared to the Fraction 3 of E2R promoter in Huh7 TWIST1 cells (Fig.7E).

\section{Discussion}

Hippo signaling was a novel and evolutionarily conserved tumor suppression pathway which was discovered to control a variety of physiological and pathological processes by attenuating cell proliferation and simultaneously promoting cell death[23]. When Hippo pathway was activated normally, MST1/2 phosphorylated LATS1/2 and consequently phosphorylated YAP1. P-YAP1 was degraded in 
cytoplasm and inactivated transcription of its downstream genes eventually. During carcinogenesis, it was found that Hippo signaling pathway was inactivated and LATS1/2 was not able to be phosphorylated anymore, thus, more YAP1 protein translocated into tumor cell nucleus where YAP1 was bound with TEAD4 and then resulted in activation of its downstream pathway. Previous study showed that Hippo-deficient liver suffered from more rapid cirrhosis and HCC development compared to control mouse models, which indicated that inactivation of Hippo signaling was involved closely in hepatocarcinogenesis[24, 25]. Several investigations revealed that YAP1 could resulted in HCC progression via TEAD4-driven activation of ERBB2/PI3K/AKT pathway[26] , HNF4a signaling[27], SERPINE1 signaling[28]. However, it remains unclear about the mechanism of inactivation of Hippo/YAP1 signaling in HCC.

In this study, we found that TEAD4, an important downstream effector of the Hippo pathway, was aberrantly over-expressed in HCC tissues compared to adjacent liver tissues. And up-regulation of TEAD4 in tumor tissues was positively associated with worse post-surgical prognosis of HCC patients. In the in vitro experiments, enforced expression of TEAD4 was verified to enhance HCC cell proliferation, whereas knockdown of TEAD4 inhibited proliferation of HCC cells. In nude mouse model, HCC xenografts driven from HCC over-expressing TEAD4 were growing dramatically faster than those from control HCC cells, which also supported that TEAD4 was critical to HCC growth. Furthermore, overexpression of TEAD4 increased migration and invasion capacities of HCC cells in vitro and in vivo via up-regulating TWIST1. And analysis of TCGA database also confirmed that there was significantly positive correlation between TEAD4 and TWIST1 at the mRNA level in HCC tissues. The mechanismic investigation on increased metastatic ability revealed that TEAD4 protein was bound with TWIST1 promoter and consequently increased its expression, which resulted in EMT phenotype of HCC cells.

GPCRs are the largest family of human cell surface receptors, which has found to contribute to a variety of physiological and pathological processes via transmitting different extracellular signals into cells[29, 30]. Recently, several studies revealed that diverse signals activated by GPCR regulated YAP/TAZ activity, positively or negatively, which was dependent on the nature of signals[31-37]. Here, we found that E2R, a kind of GPCR, was positively correlated with YAP1, TEAD4 and TWIST1 after analyzing the TCGA database, which indicated initially that E2R was involved in aberrant activation of YAP1/TEAD4/TWIST1 pathway. Because TWIST1 also mediated the transcription of various genes as a transcription factor[38, 39], we attempted to figure out whether TWIST1 regulated the expression of E2R and then activated YAP1/TEAD4 pathway. Enforced expression of TWIST1 was found here to increase the expression of E2R, YAP1 and TEAD4, while inhibiting YAP1 phosphorylation in HCC cells. Interestingly, after silencing E2R expression in HCC cells, over-expression of TWIST1 did not lead to up-regulation of TEAD4 and suppression of YAP1 phosphorylation anymore. Bio-informatic analysis showed that there were 10 potential TWIST1-binding E-boxes (CANNTG) found in the E2R promoter. As assessed by luciferase reporter assay with mutant sequences and ChIP assays, Fraction 1 (-1999 bp -1756 bp) and Fraction 2 (-1638 bp -1456 bp) of the E2R promoter were identified to be TWIST1 protein binding site and TWIST1 increased E2R expression directly via bound with its promoter. These data supported strongly that TWIST1 up-regulated by TEAD4 enhanced E2R expression, repressed Hippo/YAP1 signaling, and 
consequently positively feedback activated YAP1/TEAD4/TWIST1 pathway in HCC cells. And aberrant activation of E2R/YAP1/TEAD4/TWIST1 pathway promoted HCC progression through inducing EMT.

\section{Conclusion}

This investigation unearthed that TEAD4 was aberrantly up-regulated in HCC tissues and its overexpression in tumor tissues predicted unfavorable prognosis after surgery. TEAD4 increased TWIST1 expression via binding with its promoter and promoted HCC growth and metastasis via inducing EMT. And TWIST1 up-regulated E2R expression, which consequently activated YAP1/TEAD4/TWIST1 pathway in HCC. The evidence of the mechanistic interplay between E2R and YAP1/TEAD4/TWIST1 axis provided a novel insight for further understanding of mechanism of inactivation of Hippo/YAP1 signaling in HCC.

\section{Declarations}

Funding:The source of grant support: This study was supported by grants from National Natural Scientific Foundation of China (81301743 and 81572733 to Xin Zheng), Research Fund for the doctoral Program of High Education of China from Ministry of Education (No. 20120201120090 to Xin Zheng), New Medicine Research Project from THE First Hospital of Xian Jiaotong University (XJTU1AF-CRF-2016002), Key Science and Technology Program of Shaanxi Province (No. 2014K11-01-01-21 and 2016SF-206 to Xin Zheng) and the Fundamental Research Funds for the Basic Research Operating expenses Program of Central College sponsored by Xi'an Jiaotong University to Xin Zheng.

Conflicts of interest/Competing interests: The authors declare no potential conflicts of interest.

Availability of data and material: All data generated or analysed during this study are included in this published article.All raw data can be provided from the corresponding author on reasonable request.

Ethics approval[This investigation has been approved by the Ethical Committee of the First Affiliated Hospital of Xian Jiaotong University.

Consent to participate[One hundred and thirty HCC samples were obtained from the Department of Hepatobiliary Surgery, the First Affiliated Hospital of Xian Jiaotong University with written informed consent from the patients.

Consent to publication: All patients have signed the written informed consent for the publication of material relating to them in domestic and international academic journals.

\section{References}

1. Yang JD, Hainaut P, Gores GJ et al (2019) A global view of hepatocellular carcinoma: trends, risk, prevention and management. Nat Rev Gastroenterol Hepatol 16:589-604. 
2. European Association for the Study of the Liver. Electronic address eee, European Association for the Study of the L (2018) EASL Clinical Practice Guidelines: Management of hepatocellular carcinoma. J Hepatol 69:182-236. https://doi/org/10.1016/j.jhep.2018.03.019

3. Cersosimo RJ (2021) Systemic targeted and immunotherapy for advanced hepatocellular carcinoma. Am J Health Syst Pharm 78:187-202. https://doi/org/10.1093/ajhp/zxaa365

4. Kudo M (2020) Recent Advances in Systemic Therapy for Hepatocellular Carcinoma in an Aging Society: 2020 Update. Liver Cancer 9:640-662. https://doi/org/10.1159/000511001

5. Zhao B, Li L, Tumaneng $\mathrm{K}$ et al (2010) A coordinated phosphorylation by Lats and CK1 regulates YAP stability through SCF(beta-TRCP). Genes Dev 24:72-85. https://doi/org/10.1101/gad.1843810

6. Liu CY, Zha ZY, Zhou X et al (2010) The hippo tumor pathway promotes TAZ degradation by phosphorylating a phosphodegron and recruiting the SCF\{beta\}-TrCP E3 ligase. J Biol Chem 285:3715937169. https://doi/org/10.1074/jbc.M110.152942

7. Zanconato F, Forcato M, Battilana $G$ et al (2015) Genome-wide association between YAP/TAZ/TEAD and AP-1 at enhancers drives oncogenic growth. Nat Cell Biol 17:1218-1227. https://doi/org/10.1038/ncb3216

8. Plouffe SW, Lin KC, Moore JL, 3rd et al (2018) The Hippo pathway effector proteins YAP and TAZ have both distinct and overlapping functions in the cell. J Biol Chem 293:11230-11240.

https://doi/org/10.1074/jbc.RA118.002715

9. Zhang W, Li J, Wu Y et al (2018) TEAD4 overexpression promotes epithelial-mesenchymal transition and associates with aggressiveness and adverse prognosis in head neck squamous cell carcinoma. Cancer Cell Int 18:178. https://doi/org/10.1186/s12935-018-0675-z

10. Wu Y, Zheng Q, Li Y et al (2019) Metformin targets a YAP1-TEAD4 complex via AMPKalpha to regulate CCNE1/2 in bladder cancer cells. J Exp Clin Cancer Res 38:376. https://doi/org/10.1186/s13046-019$1346-1$

11. Kang W, Huang T, Zhou $Y$ et al (2018) miR-375 is involved in Hippo pathway by targeting YAP1/TEAD4-CTGF axis in gastric carcinogenesis. Cell Death Dis 9:92. https://doi/org/10.1038/s41419017-0134-0

12. Yu T, Song J, Zhou H et al (2021) Nuclear TEAD4 with SIX1 Overexpression is an Independent Prognostic Marker in the Stage I-III Colorectal Cancer. Cancer Manag Res 13:1581-1589. https://doi/org/10.2147/CMAR.S260790 
13. Xia Y, Chang T, Wang Y et al (2014) YAP promotes ovarian cancer cell tumorigenesis and is indicative of a poor prognosis for ovarian cancer patients. PLoS One 9:e91770.

https://doi/org/10.1371/journal.pone.0091770

14. Dent P, Booth L, Roberts JL et al (2019) Neratinib inhibits Hippo/YAP signaling, reduces mutant K-RAS expression, and kills pancreatic and blood cancer cells. Oncogene 38:5890-5904.

https://doi/org/10.1038/s41388-019-0849-8

15. Tao J, Calvisi DF, Ranganathan S et al (2014) Activation of beta-catenin and Yap1 in human hepatoblastoma and induction of hepatocarcinogenesis in mice. Gastroenterology 147:690-701. https://doi/org/10.1053/j.gastro.2014.05.004

16. Zhang T, Zhang J, You X et al (2012) Hepatitis B virus X protein modulates oncogene Yes-associated protein by CREB to promote growth of hepatoma cells. Hepatology 56:2051-2059.

https://doi/org/10.1002/hep.25899

17. Wu H, Xiao Y, Zhang S et al (2013) The Ets transcription factor GABP is a component of the hippo pathway essential for growth and antioxidant defense. Cell Rep 3:1663-1677.

https://doi/org/10.1016/j.celrep.2013.04.020

18. Misra JR, Irvine KD (2018) The Hippo Signaling Network and Its Biological Functions. Annu Rev Genet 52:65-87. https://doi/org/10.1146/annurev-genet-120417-031621

19. Bae SJ, Luo X (2018) Activation mechanisms of the Hippo kinase signaling cascade. Biosci Rep 38. https://doi/org/10.1042/BSR20171469

20. Wang C, Dou C, Wang Y et al (2019) TLX3 repressed SNAl1-induced epithelial-mesenchymal transition by directly constraining STAT3 phosphorylation and functionally sensitized 5-FU chemotherapy in hepatocellular carcinoma. Int J Biol Sci 15:1696-1711. https://doi/org/10.7150/ijbs.33844

21. Yu OM, Benitez JA, Plouffe SW et al (2018) YAP and MRTF-A, transcriptional co-activators of RhoAmediated gene expression, are critical for glioblastoma tumorigenicity. Oncogene 37:5492-5507. https://doi/org/10.1038/s41388-018-0301-5

22. Yagi $H$, Asanoma $K$, Ohgami T et al (2016) GEP oncogene promotes cell proliferation through YAP activation in ovarian cancer. Oncogene 35:4471-4480. https://doi/org/10.1038/onc.2015.505

23. Zanconato F, Cordenonsi M, Piccolo S (2016) YAP/TAZ at the Roots of Cancer. Cancer Cell 29:783803. https://doi/org/10.1016/j.ccell.2016.05.005

24. Lee KP, Lee JH, Kim TS et al (2010) The Hippo-Salvador pathway restrains hepatic oval cell proliferation, liver size, and liver tumorigenesis. Proc Natl Acad Sci U S A 107:8248-8253. https://doi/org/10.1073/pnas.0912203107 
25. Song H, Mak KK, Topol L et al (2010) Mammalian Mst1 and Mst2 kinases play essential roles in organ size control and tumor suppression. Proc Natl Acad Sci U S A 107:1431-1436. https://doi/org/10.1073/pnas.0911409107

26. Wang EY, Cheng JC, Thakur A et al (2018) YAP transcriptionally regulates ErbB2 to promote liver cell proliferation. Biochim Biophys Acta Gene Regul Mech. https://doi/org/10.1016/j.bbagrm.2018.07.004

27. Cai WY, Lin LY, Hao H et al (2017) Yes-associated protein/TEA domain family member and hepatocyte nuclear factor 4-alpha (HNF4alpha) repress reciprocally to regulate hepatocarcinogenesis in rats and mice. Hepatology 65:1206-1221. https://doi/org/10.1002/hep.28911

28. Marquard S, Thomann S, Weiler SME et al (2020) Yes-associated protein (YAP) induces a secretome phenotype and transcriptionally regulates plasminogen activator Inhibitor-1 (PAI-1) expression in hepatocarcinogenesis. Cell Commun Signal 18:166. https://doi/org/10.1186/s12964-020-00634-6

29. Hilger D, Masureel M, Kobilka BK (2018) Structure and dynamics of GPCR signaling complexes. Nat Struct Mol Biol 25:4-12. https://doi/org/10.1038/s41594-017-0011-7

30. Gundamaraju R, Lu W, Azimi I et al (2020) Endogenous Anti-Cancer Candidates in GPCR, ER Stress, and EMT. Biomedicines 8. https://doi/org/10.3390/biomedicines8100402

31. Zhou X, Wang S, Wang Z et al (2015) Estrogen regulates Hippo signaling via GPER in breast cancer. J Clin Invest 125:2123-2135. https://doi/org/10.1172/JCI79573

32. Cai H, Xu Y (2013) The role of LPA and YAP signaling in long-term migration of human ovarian cancer cells. Cell Commun Signal 11:31. https://doi/org/10.1186/1478-811X-11-31

33. Cheng JC, Wang EY, Yi Y et al (2018) S1P Stimulates Proliferation by Upregulating CTGF Expression through S1PR2-Mediated YAP Activation. Mol Cancer Res 16:1543-1555. https://doi/org/10.1158/15417786.MCR-17-0681

34. Mo JS, Yu FX, Gong R et al (2012) Regulation of the Hippo-YAP pathway by protease-activated receptors (PARs). Genes Dev 26:2138-2143. https://doi/org/10.1101/gad.197582.112

35. Zhou PJ, Xue W, Peng J et al (2017) Elevated expression of Par3 promotes prostate cancer metastasis by forming a Par3/aPKC/KIBRA complex and inactivating the hippo pathway. J Exp Clin Cancer Res 36:139. https://doi/org/10.1186/s13046-017-0609-y

36. Nag JK, Bar-Shavit R (2018) Transcriptional Landscape of PARs in Epithelial Malignancies. Int J Mol Sci 19. https://doi/org/10.3390/ijms19113451

37. Zheng $\mathrm{CH}$, Chen XM, Zhang FB et al (2018) Inhibition of CXCR4 regulates epithelial mesenchymal transition of NSCLC via the Hippo-YAP signaling pathway. Cell Biol Int 42:1386-1394. https://doi/org/10.1002/cbin.11024 
38. Qin Q, Xu Y, He T et al (2012) Normal and disease-related biological functions of Twist1 and underlying molecular mechanisms. Cell Res 22:90-106. https://doi/org/10.1038/cr.2011.144

39. Li Z, Chen B, Dong W et al (2019) MKL1 promotes endothelial-to-mesenchymal transition and liver fibrosis by activating TWIST1 transcription. Cell Death Dis 10:899. https://doi/org/10.1038/s41419-0192101-4

\section{Tables}

Table 1 The primers used in qRT-PCR assay

\begin{tabular}{lll}
\hline Gene & Forward primer $\left(5^{\prime}-3^{\prime}\right)$ & Reverse primer $\left(5^{\prime}-\mathbf{3}^{\prime}\right)$ \\
\hline GAPDH & TGACTTCAACAGCGACACCCA & CACCCTGTTGCTGTAGCCAAA \\
TEAD4 & GGCACCATTACCTCCAACGA & AGCTTGATGTAGCGGGCAAT \\
TWIST1 & GTCACGGGTAAGGACCGTTT & GTTTAGGTCTCTGCGGCTGT \\
$E 2 R$ & GGCAGTGCTGGGTATCTTCT & TGTTGAAGAGCGTTCCCCTG \\
\hline
\end{tabular}

Table 2 Demographic information and clinical features of 130 HCC patients 


\begin{tabular}{|c|c|c|c|c|c|}
\hline \multirow{2}{*}{\multicolumn{2}{|c|}{ Clinicopathological features }} & \multicolumn{2}{|c|}{ No. of Patients } & \multirow{3}{*}{$\begin{array}{c}X^{2} \\
0.001\end{array}$} & \multirow{3}{*}{$\begin{array}{c}\text { P value } \\
0.975\end{array}$} \\
\hline & & \multirow{2}{*}{$\begin{array}{c}\text { Low TEAD4 } \\
12\end{array}$} & \multirow{2}{*}{$\begin{array}{c}\text { High TEAD4 } \\
24\end{array}$} & & \\
\hline Age (years) & $<50$ & & & & \\
\hline & $\geq 50$ & 31 & 63 & & \\
\hline \multirow[t]{2}{*}{ Gender } & Male & 32 & 56 & 1.329 & 0.249 \\
\hline & Female & 11 & 31 & & \\
\hline \multirow[t]{2}{*}{ HBV infection } & Absent & 13 & 17 & 1.853 & 0.173 \\
\hline & Present & 30 & 70 & & \\
\hline \multirow[t]{2}{*}{$\operatorname{AFP}(n g / m L)$} & $<400$ & 20 & 18 & 9.276 & 0.002 \\
\hline & $\geq 400$ & 23 & 69 & & \\
\hline \multirow[t]{2}{*}{ Tumor size $(\mathrm{cm})$} & $<5$ & 30 & 40 & 6.554 & 0.001 \\
\hline & $\geq 5$ & 13 & 47 & & \\
\hline \multirow[t]{2}{*}{ Liver cirrhosis } & Absent & 5 & 8 & 0.189 & 0.664 \\
\hline & Present & 38 & 79 & & \\
\hline \multirow[t]{2}{*}{ Edmondson-Steiner Classification } & $\mathrm{I}+\mathrm{II}$ & 14 & 37 & 1.200 & 0.273 \\
\hline & III + IV & 29 & 50 & & \\
\hline \multirow[t]{2}{*}{ TNM } & $\mathrm{I}+\mathrm{II}$ & 31 & 53 & 1.571 & 0.210 \\
\hline & III + IV & 12 & 34 & & \\
\hline \multirow[t]{2}{*}{ PVTT } & Absent & 34 & 51 & 5.317 & 0.021 \\
\hline & Present & 9 & 36 & & \\
\hline \multirow[t]{2}{*}{ Tumor number } & Single & 42 & 66 & 9.738 & 0.002 \\
\hline & Multiple & 1 & 21 & & \\
\hline \multirow[t]{2}{*}{ Microvascular invasion (MVI) } & Absent & 34 & 33 & 19.499 & $<0.001$ \\
\hline & Present & 9 & 54 & & \\
\hline
\end{tabular}

Table 3 Univariate and multivariate analyses of post-surgical prognostic factors in HCC patients 


\begin{tabular}{|c|c|c|c|c|}
\hline \multirow[t]{2}{*}{ Clinicopathological features } & \multicolumn{2}{|c|}{ Univariate Analysis } & \multicolumn{2}{|c|}{ Multivariate Analysis } \\
\hline & $\mathrm{RR}(95 \% \mathrm{CI})$ & $P$ value & RR $(95 \% \mathrm{CI})$ & P value \\
\hline Age $(<50$ years $v s . \geq 50$ years $)$ & $\begin{array}{c}0.872(0.412- \\
2.123)\end{array}$ & 0.552 & $\begin{array}{c}0.592(0.219- \\
1.896)\end{array}$ & 0.593 \\
\hline Gender (Female vs. Male) & $\begin{array}{c}0.742(0.239- \\
3.416)\end{array}$ & 0.437 & $\begin{array}{c}0.539(0.254- \\
2.993)\end{array}$ & 0.215 \\
\hline HBV infection & $\begin{array}{c}1.638(0.879- \\
3.238)\end{array}$ & 0.091 & $\begin{array}{c}1.391(0.774- \\
2.989)\end{array}$ & 0.086 \\
\hline High AFP level ( $\geq 400 \mathrm{ng} / \mathrm{mL}$ ) & $\begin{array}{c}1.326(0.338- \\
2.986)\end{array}$ & 0.105 & $\begin{array}{c}1.225(0.439- \\
2.285)\end{array}$ & 0.093 \\
\hline Larger tumor size & $\begin{array}{c}1.438(0.429- \\
3.135)\end{array}$ & 0.228 & $\begin{array}{c}1.210(0.582- \\
2.878)\end{array}$ & 0.213 \\
\hline Liver cirrhosis & $\begin{array}{c}2.138(1.279- \\
4.293)\end{array}$ & 0.043 & $\begin{array}{c}1.837(1.183- \\
2.539)\end{array}$ & 0.032 \\
\hline $\begin{array}{l}\text { Advanced Edmondson-Steiner } \\
\text { Classification }\end{array}$ & $\begin{array}{c}2.533(0.792- \\
3.829)\end{array}$ & 0.112 & $\begin{array}{c}1.926(0.883- \\
3.342)\end{array}$ & 0.182 \\
\hline Advanced TNM staging & $\begin{array}{c}2.821(1.139- \\
4.302)\end{array}$ & 0.022 & $\begin{array}{c}2.640(0.972- \\
3.194)\end{array}$ & 0.110 \\
\hline PVTT & $\begin{array}{c}3.013(1.328- \\
5.323)\end{array}$ & 0.008 & $\begin{array}{c}2.319(1.311- \\
4.242)\end{array}$ & 0.010 \\
\hline Multiple tumor lesion & $\begin{array}{c}3.425(2.124- \\
5.435)\end{array}$ & $\begin{array}{c}< \\
0.001\end{array}$ & $\begin{array}{c}3.119(1.783- \\
5.029)\end{array}$ & $\begin{array}{c}< \\
0.001\end{array}$ \\
\hline MVI & $\begin{array}{c}2.902(1.509- \\
4.769)\end{array}$ & 0.003 & $\begin{array}{c}2.578(1.349- \\
4.344)\end{array}$ & 0.005 \\
\hline Higher TEAD4 in tumor tissue & $\begin{array}{l}1.837(1.110 \\
-3.805)\end{array}$ & 0.015 & $\begin{array}{c}1.634(1.038- \\
3.232)\end{array}$ & 0.017 \\
\hline
\end{tabular}

Figures 
Fig.1
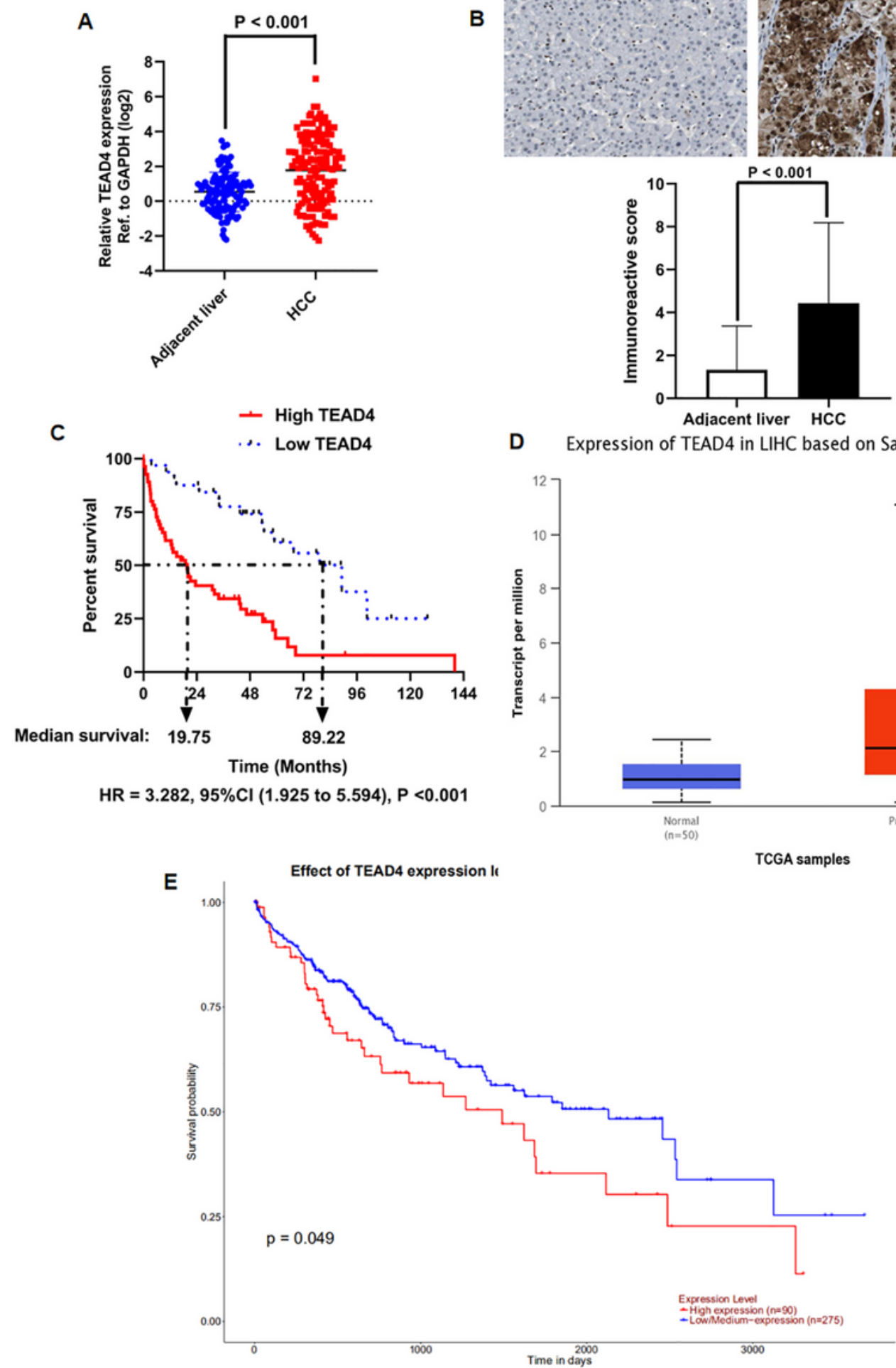

Adjacent liver HCC
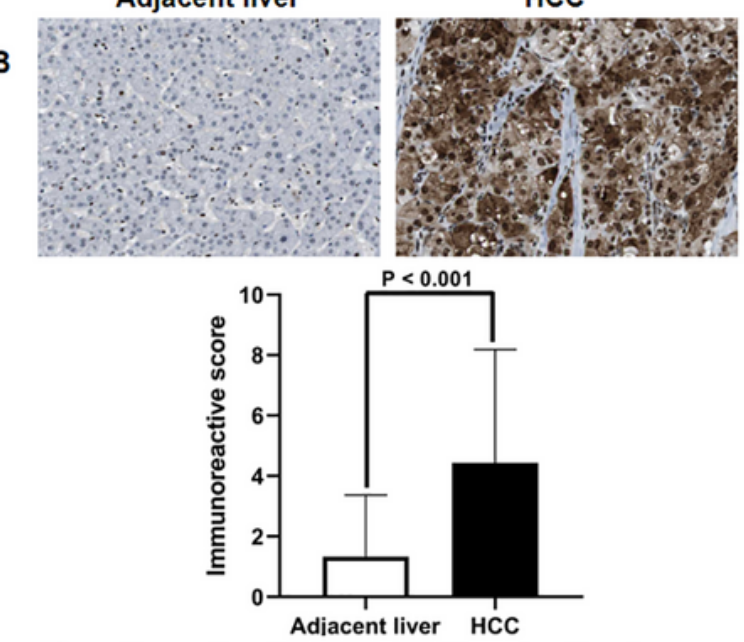

D Expression of TEAD4 in LIHC based on Sample types

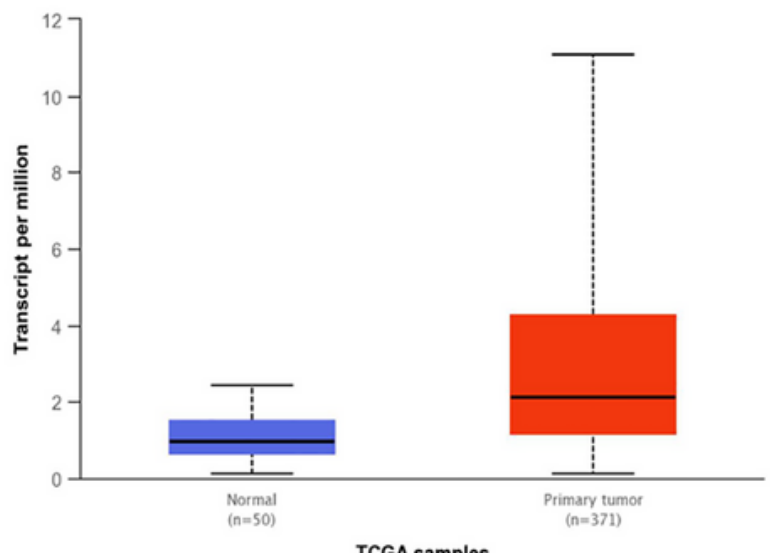

Figure 1

TEAD4 was up-regualted in HCC tissues and predicted the worse outcome after surgery. A There was significantly more TEAD4 mRNA expression in HCC tissues than adjacent liver tissues found by qRT-PCR in $130 \mathrm{HCC}$ cases. B IHC staining showed more TEAD4 protein expression in HCC tissues than adjacent liver tissues. C Comparison of Kaplan-Meier curves of 130 HCC patients by Logistic Regression analysis $(\mathrm{HR}=3.282,95 \% \mathrm{Cl}(1.925$ to 5.594$), \mathrm{P}<0.001)$. D Analysis of TCGA database with $371 \mathrm{HCC}$ samples and 
50 normal liver tissues showed that TEAD4 mRNA expression was significantly increased in HCC in contrast to normal liver tissues. E Follow-up information about $365 \mathrm{HCC}$ cases with detectable TEAD4 mRNA expression from TCGA revealed that patients with low/medium TEAD4 mRNA suffered from better post-surgery outcome compared to those with high TEAD4 mRNA expression.

Fig.2
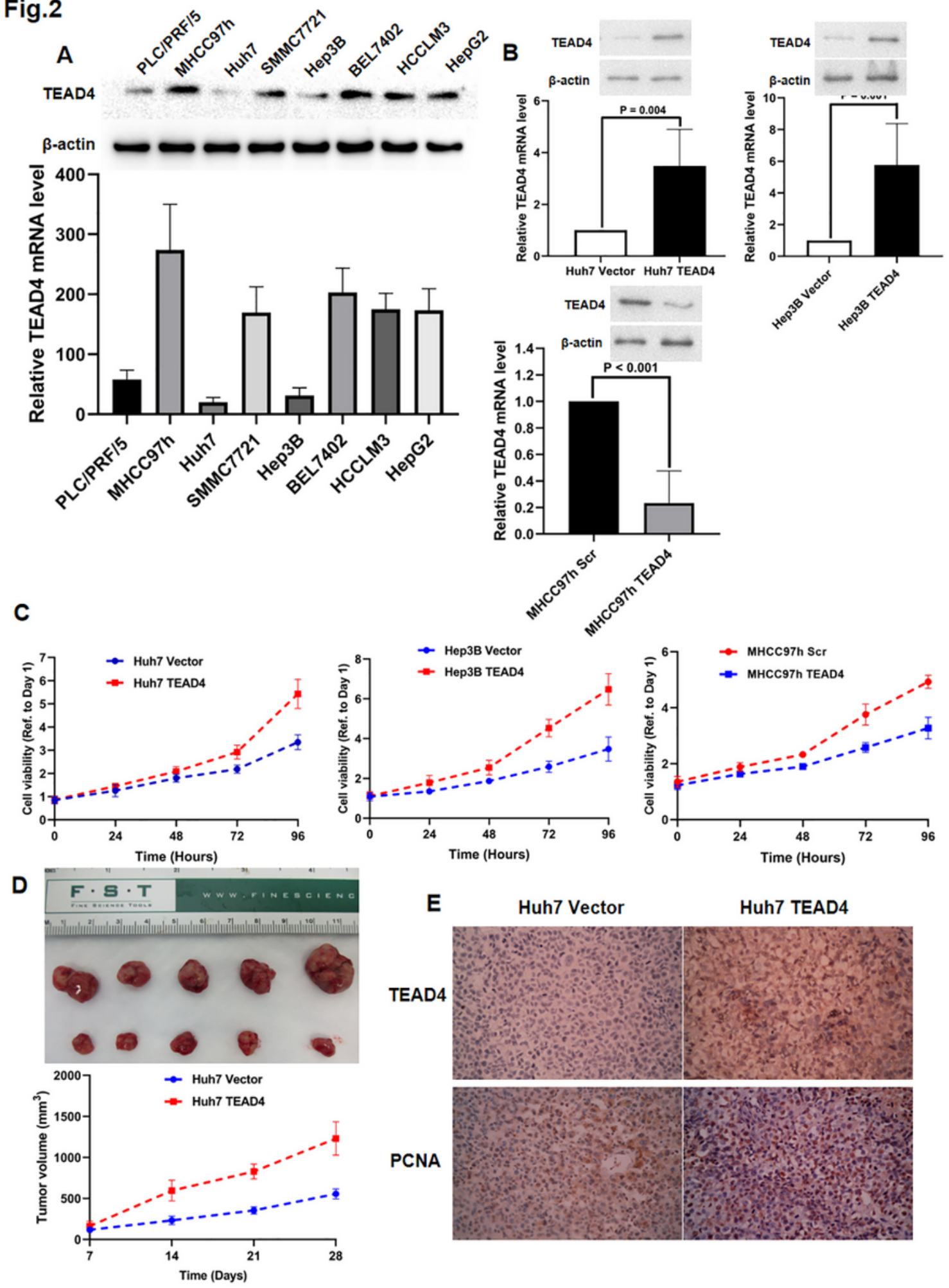

Figure 2 
Over-expression of TEAD4 promoted HCC growth in vitro and in vivo. A As assessed by both qRT-PCR and Western immunoblotting, among 7 kinds of HCC cell lines, MHCC97h cell had the highest level of TEAD4 expression at the level of both mRNA and protein, while the related low TEAD4 expression was found in both Huh7 and Hep3B cell lines. B By qRT-PCR and Western immunoblotting, it was found that transfection of TEAD4 expressing plasmid leaded to magnificent up-regulation of mRNA and protein expression of TEAD4 in both Huh7 and Hep3B cells. Transfection of TEAD4 siRNA sequences resulted in the significant decrease of TEAD4 expression in MHCC97h cells at the mRNA and protein levels. C CCK-8 assay showed that enforced expression of TEAD4 accelerated cell viability of Huh7 and Hep3B cells, whereas knockdown of TEAD4 repressed cell viability of MHCC97h cells significantly. D HCC xenografts driven from Huh7 TEAD4 cells grew faster that those from Huh7 Vector cells, which indicted that overexpression of TEAD4 enhanced HCC growth in vivo. E Analysis of HCC xenografts by IHC staining showed that there was significantly higher TEAD4 expression in HCC xenografts driven from Huh7 TEAD4 cells than those from Huh7 Vector. Meanwhile, xenografts from Huh7 TEAD4 group expressed higher PCNA than those from Huh7 Vector group, which indicated that TEAD4 overexpression promoted HCC cell proliferation in vivo. 
Fig.3

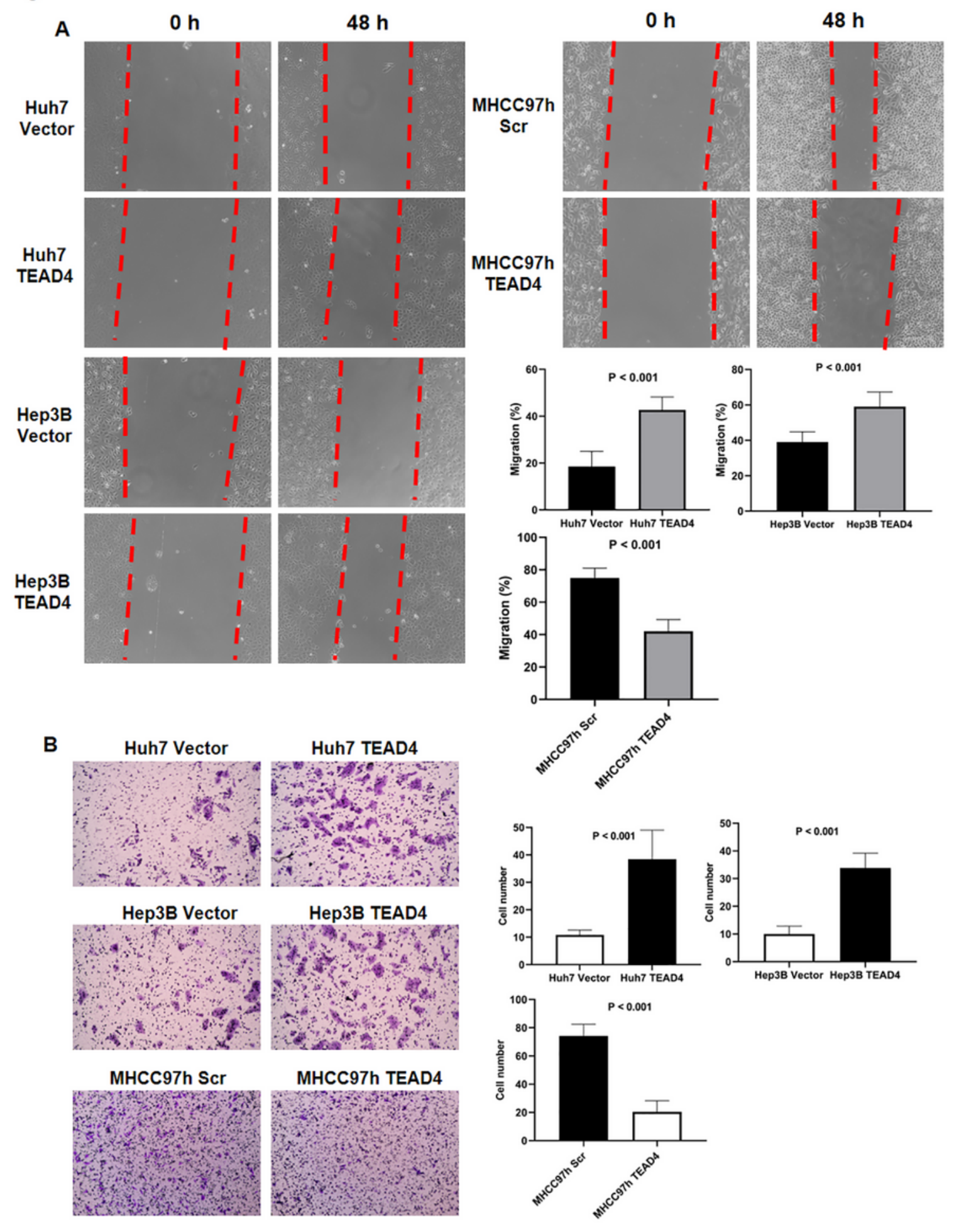

Figure 3

TEAD4 enhanced migration and invasion abilities of HCC cells. A Wound healing assay showed that overexpression of TEAD4 enforced migration capacity of both Huh7 and Hep3B cells, while silencing TEAD4 suppressed migration of MHCC97h cells. B As measured by Transwell chamber with Matrigel assay, TEAD4 over-expression enhanced invasion ability of both Huh7 and Hep3B cells, whereas knockdown of TEAD4 inhibited MHCC97h cell invasion dramatically. 
Fig.4

A

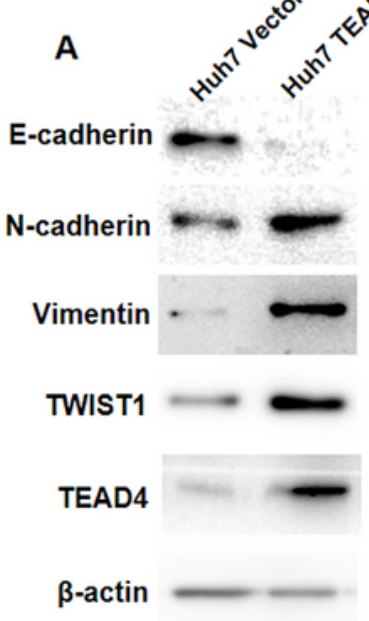

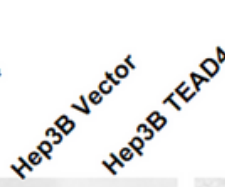
$x^{0^{30}} 2^{0^{8}}$

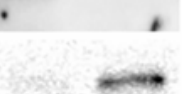

\section{-}
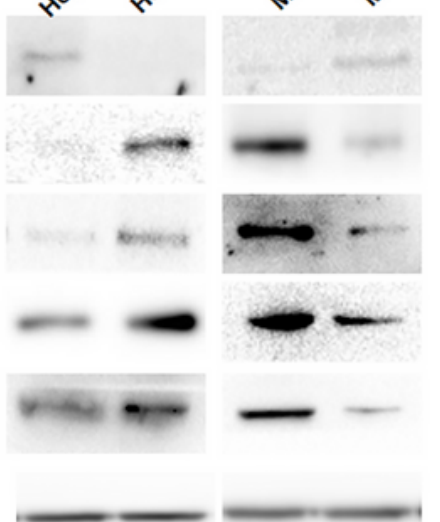
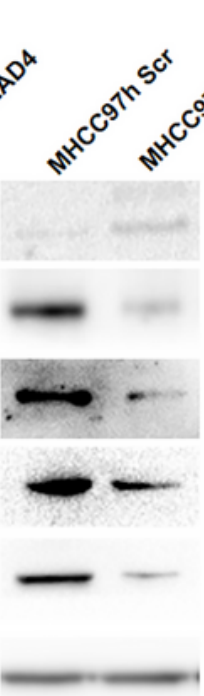

B

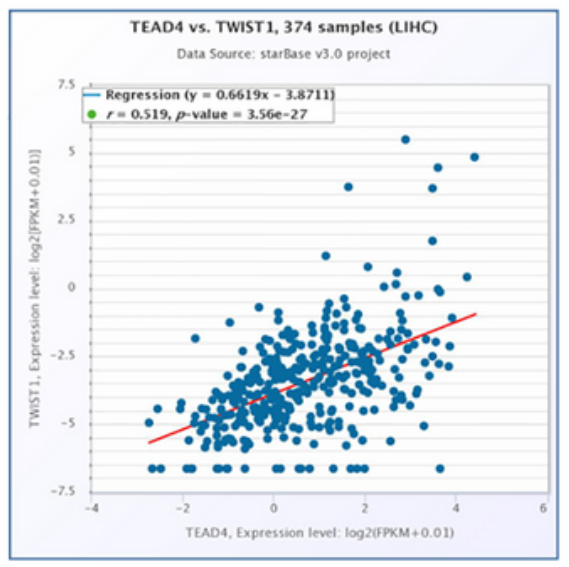

C

Huh7 Vector

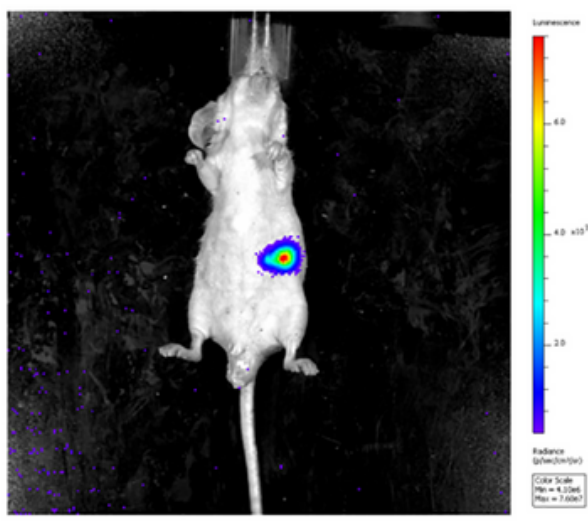

\section{Huh7 TEAD4}

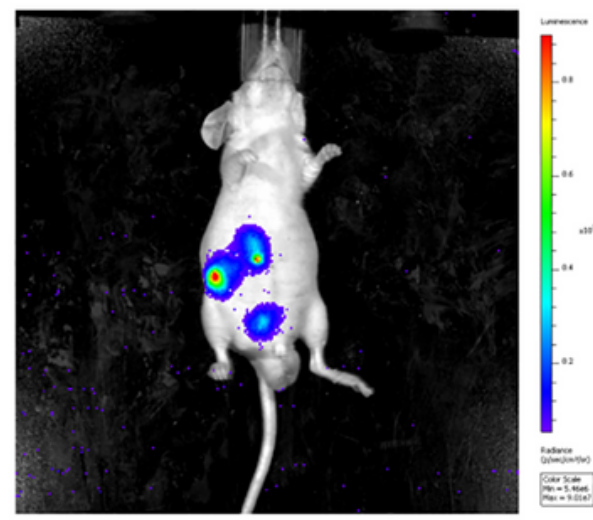

$P<0.001$
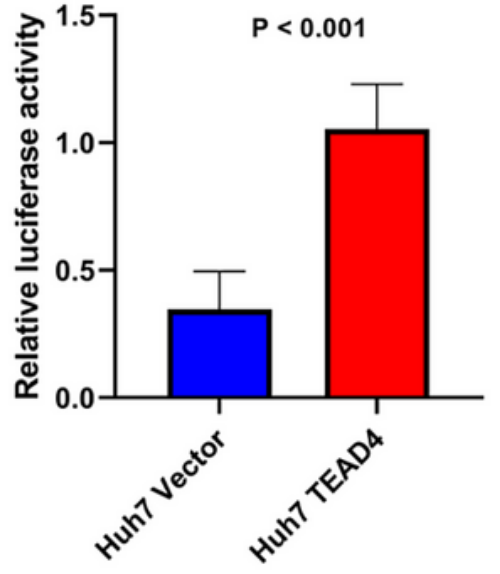

\section{Figure 4}

TEAD4 up-regulated TWIST1 and induced EMT of HCC cells. A Western immunoblotting assay showed that over-expression of TEAD4 increased the expression of TWIST1, N-cadherin and Vimentin in both Huh7 and Hep3B cells while repressing E-cadherin expression. And knockdown of TEAD4 repressed TWIST1 and mesenchymal marker (N-cadherin and Vimentin) and increased E-cadherin expression in MHCC97h cells. B Information of the TCGA database confirmed that TEAD4 mRNA expression was 
significantly associated positively with TWIST1 mRNA expression in HCC samples. C TEAD4 expressing GV492 gcGFP Lentivirus was used to establish the Huh7 cells with enhanced TEAD4 expression (Huh7 TEAD4 cells). Both Huh7 TEAD4 and Huh7 Vector cells were injected into nude mouse through tail vein. After 4 weeks, it was found by IVIS@ Lumina II system that the size of HCC xenografts from Huh7 TEAD4 group was significantly larger than those from Huh7 Vector group and more metastatic lesions were found in Huh7 TEAD4 group than Huh7 Vector group.

Fig.5

A
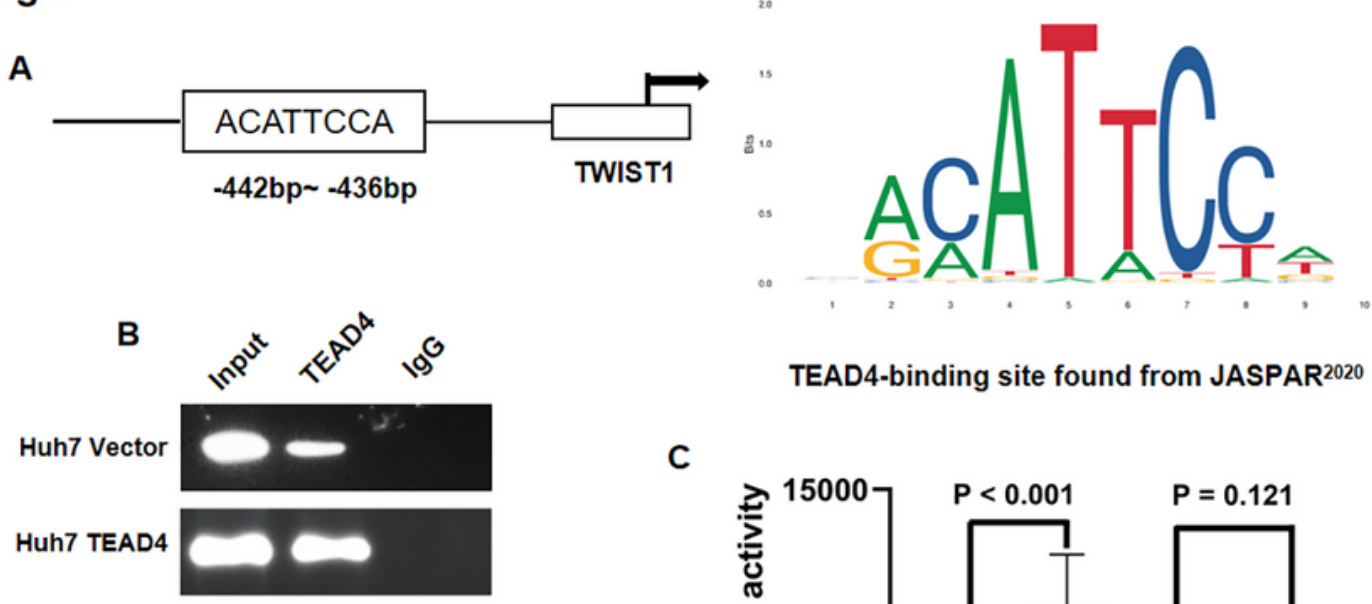

TEAD4-binding site found from JASPAR ${ }^{2020}$

C
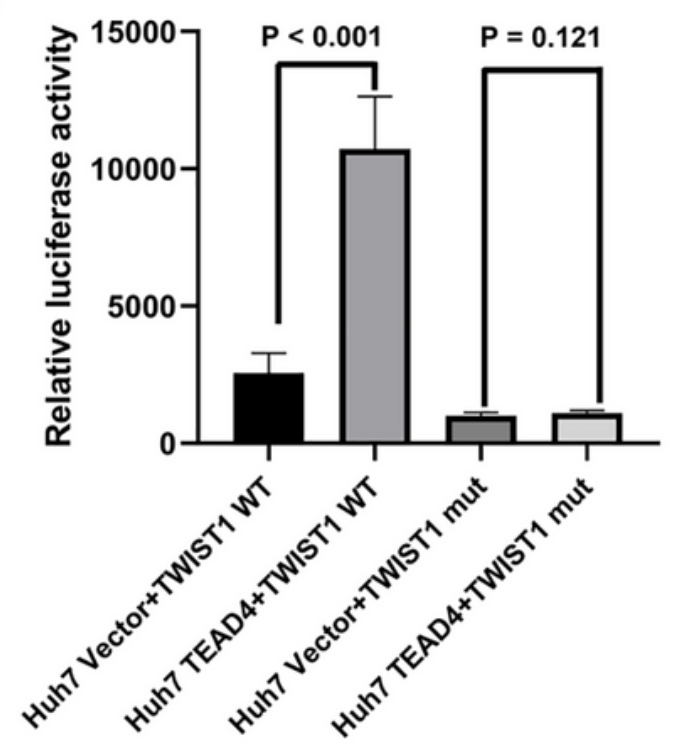

Figure 5 
TEAD4 was bound with the TWIST1 promoter and enhanced its transcription directly. A After searching JASPAR database, it was found that there was 1 potential TEAD4-binding site region. B ChIP assay confirmed that TEAD4 protein was bound with TWIST1 promoter and more fragments of the TWIST1 promoter were bound with TEAD4 protein. C Functional validation of this potential TEAD4-binding site of TWIST1 promoter using luciferase reporter assays shows that over-expression of TEAD4 enhanced the luciferase activity of the TWIST1 promoter significantly in Huh7 cells and TEAD4 over-expression was not longer able to induce the activity of the TWIST1 promoter in a reporter construct lacking the TEAD4 bindings sites. 
Fig. 6

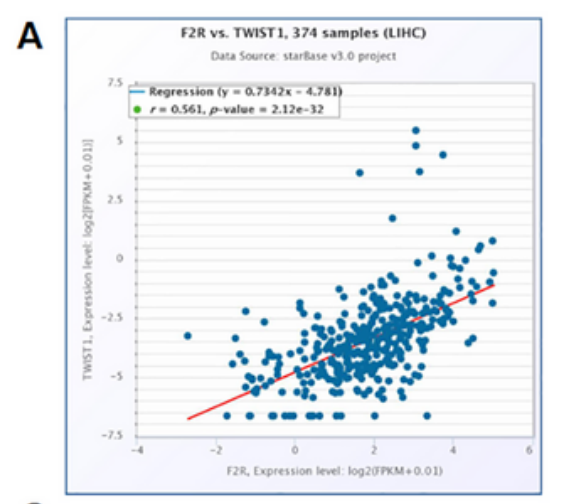

C
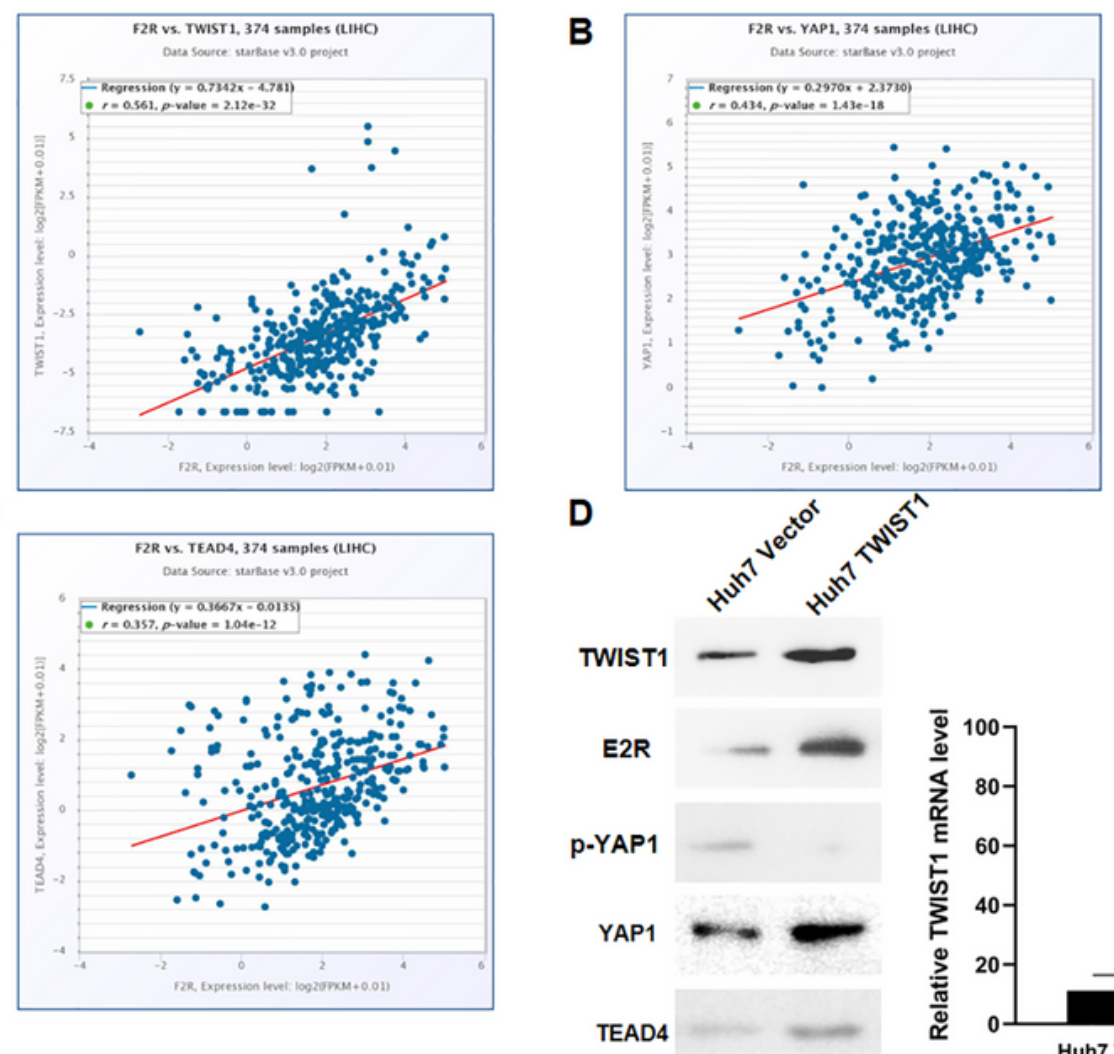

$\beta$-actin
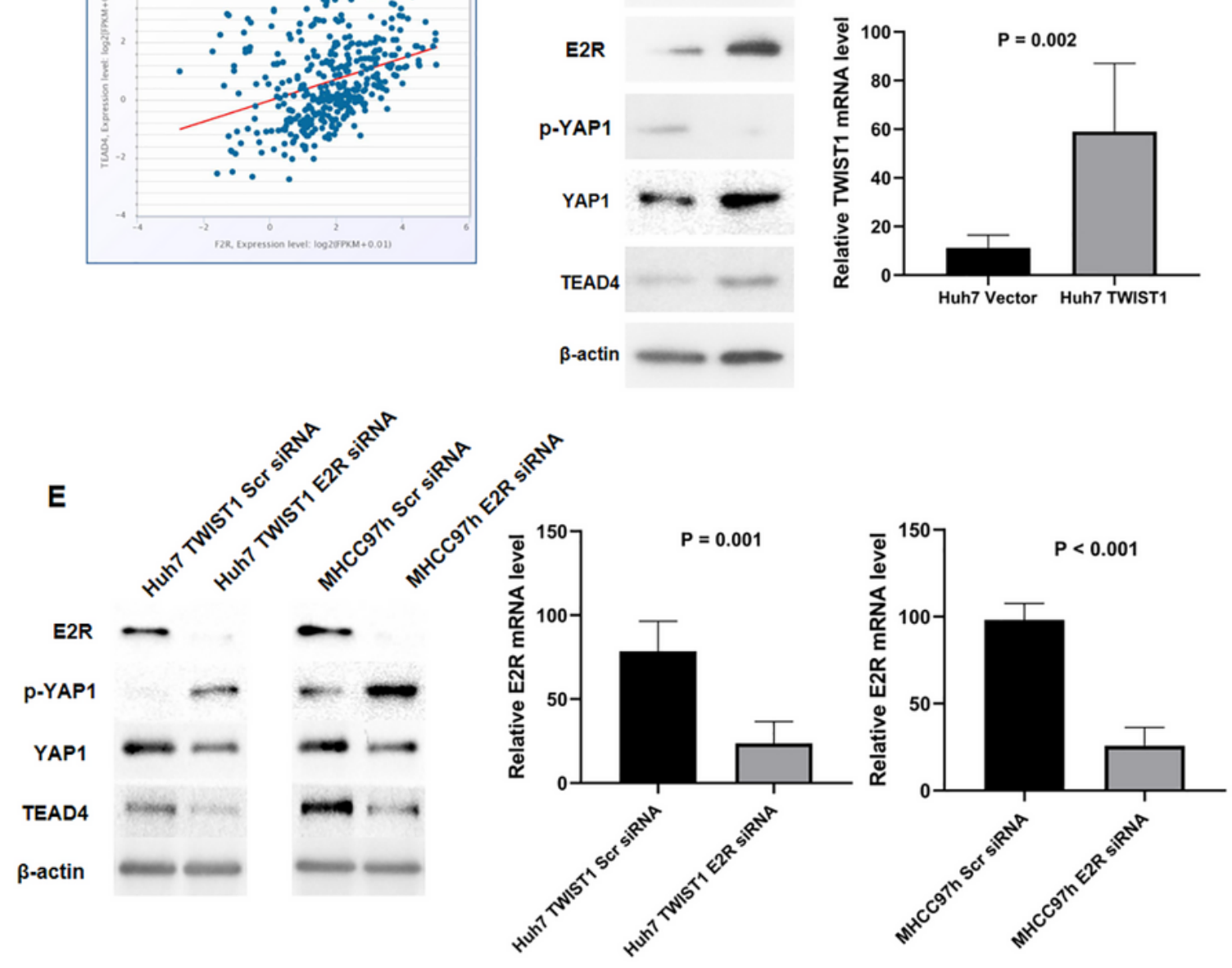

\section{Figure 6}

TWIST1 up-regulated E2R expression and activated YAP1/TEAD4 signaling in HCC cells. A Data from the TCGA database demonstrated that TWIST1 mRNA expression was magnificently correlated positively with E2R mRNA in HCC samples $(r=0.561, P<0.001)$. B Analysis of TCGA database showed that E2R mRNA was significantly associated positively with YAP1 mRNA in 374 HCC samples $(r=0.434, P<$ 0.001). C After analyzing TCGA database, it was found that E2R mRNA was dramatically related 
positively with TEAD4 mRNA in HCC samples $(r=0.357, P<0.001)$. D Both qRT-PCR and Western immunoblotting revealed that there was significantly more TWIST1 expression in Huh7 TWIST1 cells than Huh7 Vector cells. And over-expression of TWIST1 was found by Western immunobotting to increase expression of E2R, YAP1 and TEAD4, whereas decreasing YAP1 phosphorylation in Huh7 cells. E As assessed by both qRT-PCR and Western immunoblotting assay, E2R siRNA sequences silenced E2R expression in both Huh7 TWIST1 cells and MHCC97h cells. And knockdown of E2R resulted in upregulated YAP1 phosphorylation and decreased the expression of both YAP1 and TEAD4 in both Huh7 TWIST1 cells and MHCC97h cells. 
Fig.7

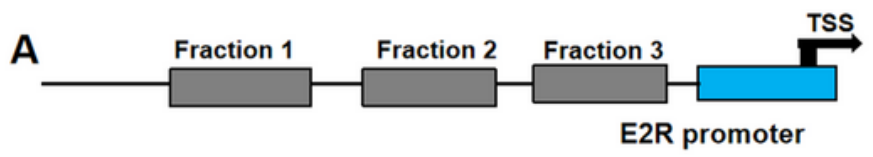

TWIST1-binding E-boxes (TBE: CANNTG)

Fraction 1:

TBE 1 CAGATG, Location: -1998 bp $\sim-1993$ bp TBE 2 CATATG, Location: -1969 bp $\sim-1964$ bp

Fraction 2: TBE 3 CAGGTG, Location: -1764 bp -1759 bp

TBE 1 CACGTG, Location: -1635 bp -1630 bp TBE 2 CAGATG, Location: -1567 bp -1562 bp

Fraction 3: TBE 3 CACATG, Location: -1635 bp $\sim-1630$ bp

TBE 1 CAGATG, Location: -1026 bp $\sim-1021$ bp TBE 2 CACTTG, Location: -666 bp -661 bp

B

TBE 3 CACCTG, Location: -433 bp $\sim-428$ bp
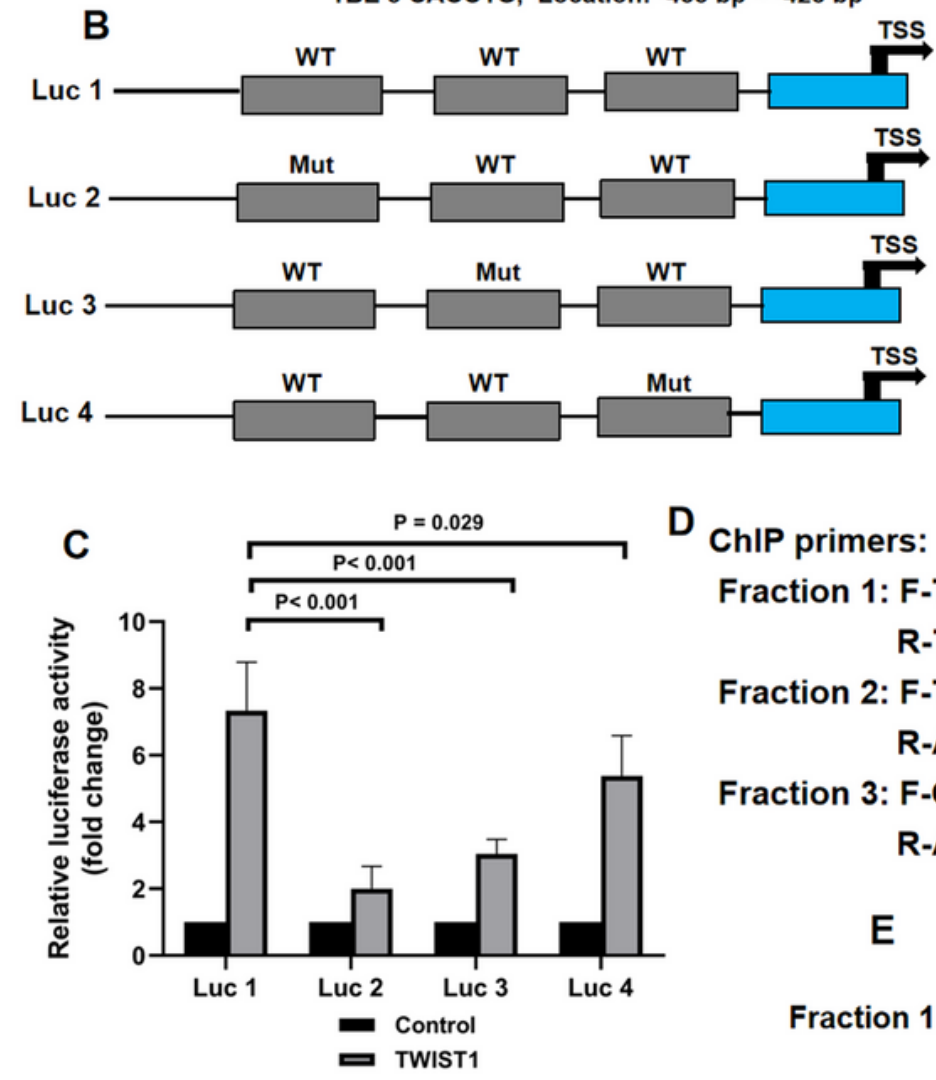

D ChIP primers:

Fraction 1: F-TGTCCGCTGTTCAGGATGTC

R-TCAGCCCATGCCCACTTTTC

Fraction 2: F-TCCTCAGACAGACACCTATAAACC

R-ACAGGCTTCTCCCCTTGTTG

Fraction 3: F-ССТCATGCACTCATCCCCAA

R-AATGGCTACTTTCAGCGCCA

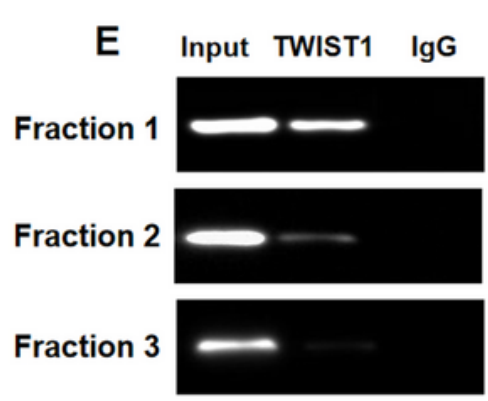

\section{Figure 7}

TWIST1 was bound with the promoter of E2R and promoted its transcription. A Bioinformatics analysis revealed that there were 10 potential TWIST1-binding E-boxes from -1346 bp to transcription start site. B Three potential TWIST1 protein-binding fractions of the E2R promoter were deleted respectively to be mutant E2R promoters. Wild type E2R promoter (Luc 1) and 3 mutant E2R promoters were cloned into Renilla luciferase vector. C As assessed by Luciferase Reporter assay, it was found that TWIST1 over- 
expression leaded to significantly more up-regulation of luciferase activity of Luc 1 and Luc 4 compared to Luc 2 and Luc 3. D The primes used in ChIP assay to detect the level of Fraction 1, 2 and 3 of E2R promoter were listed here. E ChIP assay showed that there was significantly more Fraction 1 and Fraction 2 of E2R promoter bound with TWIST1 protein than Fraction 3 in Huh7 TWIST1 cells.

\section{Supplementary Files}

This is a list of supplementary files associated with this preprint. Click to download.

- Suppl.Fig1.tif 